LBL-30747

UCB-PTH-91/21

May 1991

\title{
Duality in Gauged WZW Models
}

\author{
Elias B. Kiritsis \\ Department of Physics ${ }^{\dagger}$, University of California \\ and \\ Theoretical Physics Group \\ Lawrence Berkeley Laboratory \\ Berkeley, CA 94720
}

\begin{abstract}
All anomaly free subgroups of the internal symmetries of a WZW model are classified. It is shown that the gauging of an axial or vector U(1) subgroup provides Conformal Field Theories with target manifolds that are dual. Some implications are discussed

Published in Mod. Phys. Lett. A6 (1991) 2871.

*This work was supported in part by the Director, Office of Energy Research, Office of High Energy and Nuclear Physics, Division of High Energy Physics of the U.S. Department of Energy under Contract DE-AC03-76SF00098 and in part by the National Science Foundation under grant PHY85-15857.

${ }^{\dagger}$ e-mail addresses: KIRITSIS@LBL.bitnet, CSA::KIRITSIS, THEORY::KIRITSIS,
\end{abstract} KIRITSIS@CSA3.LBL.Gov 
Coset models constitute a class of CFT's that attracted considerable attention in recent years. The first examples were constructed long ago, [1], while a more organized effort was initiated in [2] and put forth in full generality in [3]. The models are rational CFT's and general methods exist for their exact solution, $[4,5]$. There is a Langrangian description of these models as gauged, [6] WZW models, [7]. However, the Langrangian description was hardly used to study the target geometry of the coset models, with some notable exceptions, [8, 9]

The WZW model (with group G) plays a central role in CFT. All known conformal field theories can be obtained by decomposition of its Hilbert space. Its internal symmetry is the chiral $G_{L} \otimes G_{R}$. Its world sheet symmetry is 2-d diffeomorphism and Weyl invariance. If one gauges a discrete subgroup of the internal symmetry group, orbifolds are obtained, [10]. By gauging a vector subgroup of the internal symmetry group, the coset CFT's are obtained, [6]. In general one can gauge an appropriate subgroup of its symmetries which contains internal symmetry transformations together with some worldsheet symmetries, [11]. In this way one obtains a Langrangian description of the generic affine Virasoro constructions, [12].

Another important concept, special to CFT is that of duality, [13]. It is an important symmetry of CFT and string theory. We call two target manifolds dual to each other if they give rise to the same CFT. In general, duality acts not just on the metric of the target manifold but also on other background fields. The simplest paradigm of such a symmetry is the equivalence of the theories of a free scalar field compactified on a torus with radii $R$ and $1 / R$. Such duality was observed recently in non-trivial 2-d backgrounds [14] which mimic the 4-d black hole geometry, $[15,9]$. A type of duality (also known as mirror symmetry in this case) appears in $\mathrm{N}=2$ superconformal field theories and can be very useful in solving these theories and obtain supersymmetric string vacua.

The purpose of this note is to study the existence and consequences of duality in a class of gauged WZW models at criticality. The first question 
that we will address concerns the classification of possible anomaly-free subgroups of the internal symmetry of the WZW theory, that can subsequently be gauged to obtain a conformally invariant field theory. The answer to this question is the following: in the case where the subgroup is semi-simple then it can be gauged iff it is a vector subgroup. If there are $\mathrm{U}(1)$ factors in the subgroup, then such U(1)'s can be axial or vector, while the semi-simple part has to be vector.

We will consequently focus on models where a $U(1)_{A}$ or a $U(1)_{V}$ is gauged. We will show that the two conformal theories obtained this way are dual to its other in the above-mentioned sense. We will also discuss some explicit examples and consequences.

The WZW action ${ }^{\ddagger}$ for a simple group $G$ is

$$
\begin{gathered}
I(g)=\frac{k}{16 \pi} I_{N S}(g)+\frac{i k}{24 \pi} \Gamma_{W Z}(g) \\
I_{N S}(g)=\int d^{2} x \operatorname{Tr}\left[U_{\mu} U_{\nu}\right], \Gamma_{W Z}(g)=\int_{\substack{B \\
\partial B=S^{2}}} d^{3} y \epsilon^{\mu \nu \rho} \operatorname{Tr}\left[U_{\mu} U_{\nu} U_{\rho}\right]
\end{gathered}
$$

with $U_{\mu}=g^{-1} \partial_{\mu} g, \tilde{U}_{\mu}=\partial_{\mu} g g^{-1}$. The WZ term is normalized by $\frac{1}{48 \pi^{2}} \int_{S^{3}} \operatorname{Tr}[U \wedge$ $U \wedge U] \in Z$. The model has a $G_{L} \otimes G_{R}$ invariance corresponding to left and right group transformations of $g$ with constant group elements. Since the model is critical this invariance is promoted to a local invariance giving rise to the usual current algebra, [7]. The conserved currents are

$$
\begin{aligned}
& g \rightarrow g h: J_{R}^{\mu}=\frac{k}{8 \pi} P_{-}^{\mu \nu} U_{\nu}, \quad P_{-}^{\mu \nu} \equiv \delta^{\mu \nu}-i \epsilon^{\mu \nu} \\
& g \rightarrow h g: J_{L}^{\mu}=\frac{k}{8 \pi} P_{+}^{\mu \nu} \tilde{U}_{\nu}, \quad P_{+}^{\mu \nu} \equiv \delta^{\mu \nu}+i \epsilon^{\mu \nu}
\end{aligned}
$$

\footnotetext{
${ }^{\ddagger}$ We confine ourselves to a worldsheet with the topology of $S^{2}$. The worldsheet metric is taken to be $g_{\mu \nu}=\rho \delta_{\mu \nu}$. The generalization to higher genus Riemann surfaces is similar with the additional subtlety of taking care of Gribov problems associated to noncontractible loops on the surface.
} 
A vector transformation is $g \rightarrow h g h^{-1}$ while an axial transformation is $g \rightarrow$ $h g h$.

The WZW action satisfies the Polyakov-Wiegman decomposition formula

$$
I(g h)=I(g)+I(h)-\frac{k}{8 \pi} \int d^{2} x P_{+}^{\mu \nu} \operatorname{Tr}\left[U_{\mu}(g) \tilde{U}_{\nu}(h)\right]
$$

By gauging a vector subgroup of the $G_{L} \otimes G_{R}$ symmetry one can obtain a Langrangian description of the coset models. One question we are going to investigate here is which subgroups can be gauged? We will introduce two gauge fields and try to gauge an arbitrary subgroup of the $G_{L} \otimes G_{R}$ of the theory. Thus we start from the action

$$
S_{0}(g, A, B)=I(g)+\int \operatorname{Tr}\left[A_{\mu} J_{R}^{\mu}+B_{\mu} J_{L}^{\mu}\right]-\int P_{-}^{\mu \nu} \operatorname{Tr}\left[A_{\mu} g^{-1} B_{\nu} g\right]
$$

where we added the last term for further convenience. Under the general $G_{L} \otimes G_{R}$ gauge transformation $g \rightarrow h_{1} g h_{2}$,

$$
\begin{gathered}
A_{\mu} \rightarrow A_{\mu}^{h_{2}}=h_{2}^{-1} A_{\mu} h_{2}+U_{\mu}\left(h_{2}\right), B_{\mu} \rightarrow B_{\mu}^{h_{1}}=h_{1} B_{\mu} h_{1}^{-1}+\tilde{U}_{\mu}\left(h_{1}\right) \\
S_{0}\left(h_{1} g h_{2}, A^{h_{2}}, B^{h_{1}}\right)-S_{0}(g, A, B)=\frac{i k}{24 \pi}\left[\Gamma_{W Z}\left(h_{1}\right)+\Gamma_{W Z}\left(h_{2}\right)\right]+ \\
+\frac{k}{16 \pi} \int d^{2} x \operatorname{Tr}\left[U_{\mu}\left(h_{1}\right) U^{\mu}\left(h_{1}\right)+U_{\mu}\left(h_{2}\right) U^{\mu}\left(h_{2}\right)+2 P_{+}^{\mu \nu}\left(\tilde{U}_{\mu}\left(h_{2}\right) A_{\nu}+U_{\nu}\left(h_{1}\right) B_{\mu}\right)\right]
\end{gathered}
$$

¿From (8) it is obvious that the variation contains non-local contributions due to the WZ terms which cannot be canceled by adding local functionals of the gauge fields. Thus the gauged subgroup must be such that $\Gamma_{W Z}\left(h_{1}\right)+$ $\Gamma_{W Z}\left(h_{2}\right)=0$. The solution to the previous equation is $h_{1}=\Lambda_{1} h, h_{2}=\Lambda_{2} h^{-1}$, where $\mathrm{h}$ belongs to an arbitrary subgroup $H$ and $\Lambda_{1,2}$ are elements of some (not necessarily the same) $U(1)^{n}$ subgroup of $G$ that commutes with $H$. Since we know the answer for the vector gauging we will assume that $h=1$. Then

$\delta S_{0}=\frac{k}{16 \pi} \int d^{2} x \operatorname{Tr}\left[U_{\mu}\left(\Lambda_{1}\right) U^{\mu}\left(\Lambda_{1}\right)+U_{\mu}\left(\Lambda_{2}\right) U^{\mu}\left(\Lambda_{2}\right)+2 P_{+}^{\mu \nu}\left(U_{\mu}\left(\Lambda_{2}\right) A_{\nu}+U_{\nu}\left(\Lambda_{1}\right) B_{\mu}\right)\right]$ 
The only way to cancel the terms in (9) linear in the gauge fields is to take $\Lambda_{1}=\lambda_{1} \lambda_{2}, \Lambda_{2}=\lambda_{1} \lambda_{2}^{-1}$. Thus the most general gauge group is a vector one plus an axial one in the case of $U(1)^{\prime}$ s. We have then,

$$
\begin{aligned}
& S_{V}(g, A)=I(g)+\frac{k}{8 \pi} \int d^{2} x \operatorname{Tr}\left[\left(J_{R}^{\mu}-J_{L}^{\mu}\right) A_{\mu}+P_{+}^{\mu \nu} A_{\mu} g A_{\nu} g^{-1}-A_{\mu} A^{\mu}\right] \\
& S_{A}(g, A)=I(g)+\frac{k}{8 \pi} \int d^{2} x \operatorname{Tr}\left[\left(J_{R}^{\mu}+J_{L}^{\mu}\right) A_{\mu}-P_{+}^{\mu \nu} A_{\mu} g A_{\nu} g^{-1}-A_{\mu} A^{\mu}\right]
\end{aligned}
$$

We should mention at this point that the results above hold off-criticality. The WZW model still has the $G_{L} \otimes G_{R}$ symmetry and one can gauge it. The anomaly-free subgroups are the same as in the critical case. Gauged WZW models off-criticality are candidates for off-critical integrable perturbations of coset CFT's ${ }^{\S}$

When the gauged subgroup $H$ is $\mathrm{U}(1)$, the natural question is what is the relation between the axial and the vector gauged WZW model? CFT results tell us that there is a single $G / U(1)$ coset for every distinct (group theoretically) embedding of $U(1)$ in $G$. The simple duality $R \rightarrow 1 / R$ of the toroidal theory can be implemented in the Hilbert space by changing the sign of (only) the left-handed current. Thus the naive expectation is that the axially-gauged WZW model is dual to the vectorially-gauged WZW model. However, the two actions $(10,11)$ differ by more than a flip in the sign of the left current. Despite all these contradicting signals, we will show below that the naive expectation is true: the axial and the vector theories are dual.

In order to do this we will need to derive a basic "duality transformation formula" on a free compact scalar field coupled to an external current, (see for example, [16]). Consider the field $\phi \in[0,2 \pi)$ and the partition function

$$
Z_{R}=\int_{0}^{2 \pi}[R d \phi] \exp \left[-\frac{R^{2}}{4 \pi} \int \partial_{\mu} \phi \partial^{\mu} \phi+\int \partial_{\mu} \phi J^{\mu}\right]
$$

\footnotetext{
${ }^{\S}$ Results in this direction will be reported elsewere.
} 
where $J^{\mu}$ is independent of $\phi$. We will perform a duality transformation by using the formula

$$
e^{-a b^{2}}=\frac{1}{2 \sqrt{\pi a}} \int_{-\infty}^{+\infty} d x e^{-\frac{x^{2}}{4 a}+i b x}
$$

We obtain

$$
Z_{R}=\int_{0}^{2 \pi}[R d \phi] \int\left[\frac{d B_{\mu}}{R^{2}}\right] \exp \left[-\frac{\pi}{R^{2}} \int B_{\mu} B^{\mu}+i \int B_{\mu}\left(\partial^{\mu} \phi-\frac{2 \pi}{R^{2}} J^{\mu}\right)+\frac{\pi}{R^{2}} \int J_{\mu} J^{\mu}\right]
$$

By changing variables to $B_{\mu}=\epsilon_{\mu \nu} A^{\nu}$ and doing the integral over $\phi$ we obtain

$$
Z_{R}=\int\left[d A_{\mu}\right] \frac{2 \pi}{R} \delta(F(A)) \exp \left[-\frac{\pi}{R^{2}} \int A_{\mu}^{2}-\frac{2 \pi i}{R^{2}} \int \epsilon^{\mu \nu} J_{\mu} A_{\nu}+\frac{\pi}{R^{2}} \int J_{\mu}^{2}\right]
$$

where $F(A)=\epsilon^{\mu \nu} \partial_{\mu} A_{\nu}$. The original theory was invariant under translations of $\phi$ by a constant. This implies that $\int_{S^{2}} F(A)=0$. We will now solve the $\delta$-function constraint by $A_{\mu}=\frac{1}{2 \pi} \partial_{\mu} \phi$ (the Jacobian for this is trivial).

$$
Z_{R}=\int_{0}^{2 \pi}\left[\frac{d \phi}{R}\right] \exp \left[-\frac{1}{4 \pi R^{2}} \int \partial_{\mu} \phi \partial^{\mu} \phi-\frac{i}{R^{2}} \int \epsilon^{\mu \nu} J_{\mu} \partial_{\nu} \phi+\frac{\pi}{R^{2}} \int J_{\mu} J^{\mu}\right]
$$

For $J^{\mu}=0$ (16) gives the usual $R \rightarrow 1 / R$ duality of the free scalar field theory.

Let us now consider a simple group $G$ and choose a U(1) subgroup element $Q_{\phi}=\exp \left[i \phi T_{0}\right], T_{0}$ is the generator of the $U(1)$ normalized as $\operatorname{Tr}\left[T_{0}^{2}\right]=1$. Parametrize $G \ni g=Q_{\phi} h$. Using (5) we can evaluate the WZW action

$$
I(g)=I(h)+\frac{k}{16 \pi} \int \partial_{\mu} \phi \partial^{\mu} \phi-\frac{i k}{8 \pi} \int P_{+}^{\mu \nu} \partial_{\mu} \phi \tilde{U}_{\nu}^{0}(h)
$$

where $\tilde{U}_{\mu}^{0} \equiv \operatorname{Tr}\left[T_{0} \tilde{U}_{\mu}(h)\right]$. The left-handed and right-handed U(1) currents are given by

$$
J_{R}^{\mu}=\frac{k}{2 \pi} P_{-}^{\mu \nu}\left(i M \partial_{\nu} \phi+U_{\nu}^{0}\right), J_{L}^{\mu}=\frac{k}{8 \pi} P_{+}^{\mu \nu}\left(i \partial_{\nu} \phi+\tilde{U}_{\nu}^{0}\right)
$$


where $M \equiv \operatorname{Tr}\left[T_{0} h T_{0} h^{-1}\right]$. The partition function is

$$
Z_{G}=\int\left[\frac{d g}{U(1)}\right] \int_{0}^{2 \pi}[d \phi]\left[\frac{k}{4}\right]^{\operatorname{dim} G / 2} \exp [-I(g)]
$$

where $[d g / U(1)]$ is the Haar measure restricted in the coset. By applying (16) we can obtain its dual form

$$
Z_{G}=\int\left[\frac{d g}{U(1)}\right] \int_{0}^{2 \pi}[d \phi]\left[\frac{k}{4}\right]^{\frac{(d i m G-2)}{2}} \exp \left[-I^{d u a l}(g)\right]
$$

with

$$
I^{d u a l}(g)=I(h)+\frac{1}{\pi k} \int\left(\partial_{\mu} \phi\right)^{2}-\frac{i}{2 \pi} \int P_{+}^{\mu \nu} \partial_{\mu} \phi \tilde{U}_{\nu}^{0}
$$

The left and right $\mathrm{U}(1)$ currents in the dual theory are given by

$$
\tilde{J}_{R}^{\mu}=\frac{k}{2 \pi} P_{-}^{\mu \nu}\left(\frac{4 i}{k} M \partial_{\nu} \phi+U_{\nu}^{0}\right), \tilde{J}_{L}^{\mu}=\frac{k}{8 \pi} P_{+}^{\mu \nu}\left(\frac{4 i}{k} \partial_{\nu} \phi+\tilde{U}_{\nu}^{0}\right)
$$

Consider now the vector $\mathrm{U}(1)$ gauged action

$$
S_{V}=I(g)+\frac{i k}{8 \pi} \int A_{\mu}\left(P_{-}^{\mu \nu}\left(i M \partial_{\nu} \phi+U_{\nu}^{0}\right)-P_{+}^{\mu \nu}\left(i \partial_{\nu} \phi+\tilde{U}_{\nu}^{0}\right)\right)+\frac{k}{8 \pi} \int(1+M) A_{\mu} A^{\mu}
$$

Applying (16) to (23) we obtain

$$
\int_{0}^{2 \pi}[d \phi]\left[\frac{k}{4}\right]^{\operatorname{dim} G / 2} e^{-S_{V}}=\int_{0}^{2 \pi}[d \phi]\left[\frac{k}{4}\right]^{\frac{\operatorname{dim} G-2}{2}} e^{-S_{V}^{\text {dual }}}
$$

where

$$
S_{V}^{\text {dual }}=I^{\text {dual }}(g)+\frac{i k}{8 \pi} \int A_{\mu}\left(\tilde{J}_{R}^{\mu}+\tilde{J}_{L}^{\mu}\right)+\frac{k}{8 \pi} \int(1+M) A_{\mu} A^{\mu}
$$

Inspection on (25) shows that this is the axially gauged dual theory. This proves our previous claim: The $\mathrm{U}(1)$ vector-gauged WZW model is dual to the axially-gauged one. 
We will illustrate the above by considering as an explicit example the case $G=S U(2), H=U(1)$ which represents the $Z_{k}$ parafermionic CFT. We will parametrize $\mathrm{g}$ by the Euler angles

$$
g=\exp \left(i \frac{\alpha}{2} \sigma_{3}\right) \exp \left(i \frac{\beta}{2} \sigma_{1}\right) \exp \left(i \frac{\gamma}{2} \sigma_{3}\right)
$$

where $\alpha \in[0,2 \pi), \gamma \in[0,2 \pi), \beta \in D \equiv[0, \pi] \cup[2 \pi, 3 \pi]$. The Haar measure is, $\int d \Omega=\frac{1}{16 \pi^{2}} \int_{D} \sin \beta d \beta \int_{0}^{2 \pi} d \alpha \int_{0}^{2 \pi} d \gamma$, normalized so that the group volume is one.

$$
I_{S U(2)}=\frac{k}{16 \pi} \int\left[\partial_{\mu} \alpha \partial^{\mu} \alpha+\partial_{\mu} \beta \partial^{\mu} \beta+\partial_{\mu} \gamma \partial^{\mu} \gamma+2 \cos \beta P_{+}^{\mu \nu} \partial_{\mu} \alpha \partial_{\nu} \gamma\right]
$$

with $k \in Z$. When we gauge the axial $\mathrm{U}(1)$ generated by $\sigma_{3}$ we obtain

$$
S_{A}=I_{S U(2)}+\frac{i k}{8 \pi} \int A_{\mu}\left(P_{-}^{\mu \nu} U_{\nu}+P_{+}^{\mu \nu} \tilde{U}_{\nu}\right)+\int A_{\mu} A^{\mu}(1+\cos \beta)
$$

with $U_{\mu}=-i\left(\partial_{m} \gamma+\cos \beta \partial_{\mu} \alpha\right), \tilde{U}_{\mu}=-i\left(\partial_{\mu} \alpha+\cos \beta \partial_{\mu} \gamma\right)$. The gauge transformations are $\alpha \rightarrow \alpha+\epsilon, \beta \rightarrow \beta, \gamma \rightarrow \gamma+\epsilon, A_{\mu} \rightarrow A_{\mu}-\partial_{\mu} \epsilon$. We choose the gauge fixing condition $\alpha+\gamma=2 \pi$ which has a trivial FP determinant. Performing the integral over the gauge field we obtain

$$
Z_{S U(2) / U(1)}^{A}=\int_{D}[\sin \beta d \beta] \int_{0}^{2 \pi}[d \alpha] \operatorname{det}\left[\frac{-2 \pi^{2}}{k(1+\cos \beta)}\right] e^{-S_{A}^{g . f .}}
$$

with

$$
S_{A}^{g . f .}=\frac{k}{16 \pi} \int\left[\partial_{\mu} \beta \partial^{\mu} \beta+4 \tan ^{2} \frac{\beta}{2} \partial_{\mu} \alpha \partial^{\mu} \alpha\right]
$$

We can regulate the logarithm of the determinant using $e^{-\frac{\nabla^{2}}{m^{2}}}$ where $m$ is a regulator mass. The short distance expansion of the heat kernel is

$$
\left\langle x\left|e^{-\frac{\nabla^{2}}{m^{2}}}\right| x>=\frac{m^{2}}{4 \pi}+\frac{R^{(2)}}{12 \pi}+\mathcal{O}\left(m^{2}\right)\right.
$$

"In gauged WZW models any two admissible "unitary gauge" fixings (that is a gauge fixing that does not involve the gauge field) generate target manifold metrics that are related by a reparametrization. The ratio of the FP determinants when regulated corrects the dilaton field in such a way that it provides the correct volume form on the target manifold when combined with the measure. 
where $R^{(2)}$ is the 2 -d scalar curvature. We can easily compute (see for example the second ref. [13]) by throwing away the singular piece as $m^{2} \rightarrow \infty$, that

$$
\operatorname{det}\left[\frac{-2 \pi^{2}}{k(1+\cos \beta)}\right]_{r e g}=\exp \left[-\frac{1}{8 \pi} \int d^{2} x \sqrt{g} R^{(2)} \log (1+\cos \beta)+\text { constant }\right]
$$

which provides the correct dilaton field (to one-loop order). Notice that the zero mode part of the dilaton field combined with the measure provides the correct volume element for the spacetime metric in (30). Both the metric $G_{\alpha \alpha}^{(1)}=4 \tan ^{2} \frac{\beta}{2}$ and the dilaton $\Phi^{(1)}=\frac{1}{2} \log (1+\cos \beta)$ can in principle receive corrections at higher loop order. However they always have to provide the correct volume element of the manifold. This gives the following relation between the exact metric $G_{\alpha \alpha}$ and the exact dilaton $\Phi$

$$
\Phi(\beta)=\log \left(\frac{\sin \beta}{\sqrt{G_{\alpha \alpha}}}\right)
$$

If instead we compute the vector-gauged effective action we obtain

$$
\tilde{G}_{\alpha \alpha}=4 \cot ^{2} \frac{\beta}{2}, \quad \tilde{\Phi}=\frac{1}{2} \log (1-\cos \beta)
$$

in accordance with the general argument. However it easy to see that here the dual metric is related by a reparametrization $(\beta \rightarrow \pi-\beta)$ to the original one, so the model is self-dual.

The spacetime described by the metric in (30) looks like two "bells" stuck together. Its scalar curvature is given by

$$
R=-\frac{2}{1+\cos \beta}
$$

which has a singularity along the common rim of the "bells". This is precisely where the quadratic term of the gauge field vanishes. The model has a $Z_{2}$ symmetry that interchanges the two "bells". If one mods out by that symmetry one obtains the $S O(3) / U(1)$ theory. This can be easily seen since 
the $\mathrm{SO}(3)$ manifold is obtained from the $\mathrm{SU}(2)$ one, in the parametrization (26) by restricting $\beta \in[0, \pi]$. The $S O(3) / U(1)$ theory is unitary only when $k \in 2 Z$. There is a continuous vector $\mathrm{U}(1)$ symmetry generated by constant shifts of the field $\alpha$. This is identified with the $\mathrm{U}(1)$ symmetry responsible for the conservation of the magnetic quantum number of the parafermionic primary fields in CFT. The $Z_{k}$ symmetry of the parafermion model corresponds to $\alpha \rightarrow \alpha+\frac{2 \pi}{k}$. Orbifoldizing by a discrete subgroup of that symmetry leaves the model invariant. One could also gauge this symmetry. Then after integrating out the gauge fields the resulting determinant cancels the contributions of the dilaton and the measure and one is left with a $Z_{2}$ orbifold of the scalar field $\beta$ at a radius $R=\sqrt{k}$. The same procedure aplied to the $\mathrm{SO}(3) / \mathrm{U}(1)$ coset leads to a toroidal theory with $R=\sqrt{k} / 4$.

Let us also examine the case of $S L(2, R) / U(1)$ that has attracted some attention recently, $[9,14,17]$. We will parametrize an element $g$ of $\mathrm{SL}(2, \mathrm{R})$ as

$$
g=e^{i \frac{\phi}{2} \sigma_{2}} e^{\frac{r}{2} \sigma_{1}} e^{i \frac{\psi}{2} \sigma_{2}}
$$

where $r \in[0,+\infty), \phi \in[-2 \pi, 2 \pi], \psi \in[0,2 \pi)$. The WZW action is

$$
I_{S L(2, R)}=\frac{k}{32 \pi} \int\left[\partial_{\mu} \phi \partial^{\mu} \phi+\partial_{\mu} \psi \partial^{\mu} \psi+\partial_{\mu} r \partial^{\mu} r+2 \cosh r P_{+}^{\mu \nu} \partial_{\mu} \phi \partial_{\nu} \psi\right]
$$

and the Haar measure on $S L(2, R)$ is $\sinh r d r d \phi d \psi$. We gauge the axial $\mathrm{U}(1)$ generated by $\sigma_{2}$, we gauge fix, $\phi+\psi=2 \pi$ and following the same procedure as in $\mathrm{SU}(2)$ we obtain

$$
Z_{S L(2, R) / U(1)}^{A}=\int_{0}^{+\infty}[\sinh r d r] \int_{0}^{2 \pi}[d \phi] \operatorname{det}\left[\frac{4 \pi^{2}}{k(1+\cosh r)}\right] e^{-S_{A}^{g . f} .}
$$

with

$$
S_{A}^{g . f .}=\frac{k}{32 \pi} \int\left[\partial_{\mu} r \partial^{\mu} r+4 \tanh ^{2} \frac{r}{2} \partial_{\mu} \phi \partial^{\mu} \phi\right]
$$

The determinant, via regularization gives the dilaton term $\exp \left[-\frac{1}{4 \pi} \int R^{(2)} \Phi\right]$ with $\Phi=\frac{1}{2} \log (1+\cosh r)$. 
Gauging the vector subgroup we obtain the inverse metric $\tilde{G}_{\phi \phi}=4 \operatorname{coth}^{2} \frac{r}{2}$ and dilaton $\tilde{\Phi}=\frac{1}{2} \log (1-\cosh r)$. In this case the dual manifolds are different, and the statement of duality non-trivial. As in the $\mathrm{SU}(2)$ case there is a $\mathrm{U}(1)$ symmetry in the model which when gauged will give rise to a 1-d non-compact $Z_{2}$ orbifold (the real line, moded out by $x \rightarrow-x$ ).

The duality transformations we have discussed can also be applied offcriticality. In that case we do not expect an equivalence of the two theories, but a correspondence that permutes the operator content of the theories. It is obvious from the above that such duality transformations should exist for integrable perturbations of $G / U(1)$ coset models.

To summarize, we have investigated the anomaly free subgroups of the WZW theory. Any such subgroup has to be vector unless it is a $U(1)$ in which case it can be either vector or axial vector. We then showed that the vector or axial gauging of a U(1) subgroup produces dual theories. It would be interesting to apply these results to the case of $N=2$ superconformal models, in order to shed some light into the spacetime picture of mirror symmetry.

\section{Acknowledgements}

I would like to thank O. Alvarez, K. Bardakci, M. Crescimanno, M. Douglas and A. Giveon for discussions.

\section{NOTE ADDED}

After the completion of this work we have received ref. [18] which partially overlaps with our present treatment. 


\section{References}

[1] K. Bardakci, M. Halpern, Phys. Rev. D3 (1971) 2493; M. Halpern, Phys. Rev. D4 (1971) 2398.

[2] P. Goddard, A. Kent, D. Olive, Phys. Lett. B152 (1985) 88.

[3] M. Douglas, PhD Thesis, Caltech 1988, (unpublished); F. A. Bais, P. Bouwknegt, M. Surridge, K. Schoutens, Nucl. Phys. B304 (1988) 348, 371.

[4] Vl. S. Dotsenko, V. A. Fateev, Nucl. Phys. B240[FS12] (1984) 312; ibid. B251[FS13] (1985) 691.

[5] S. Mathur, S. Mukhi, A. Sen, Nucl. Phys. B312 (1989) 15; B. Blok, S. Yankielowicz, Nucl. Phys. B321 (1989) 717; E. Kiritsis, Nucl. Phys. $\underline{\text { B329 }}$ (1990) 591.

[6] Y. S. Wu, Phys. Lett. $\underline{B 153}$ (1985) 70; W. Nahm, Duke Math. J., $\underline{54}$ (1987) 579; K. Bardakci, E. Rabinovici, B. Säring, Nucl. Phys. B299 (1988) 157; D. Altschuler, K. Bardakci, E. Rabinovici, Comm. Math. Phys. 118 (1988) 241; K. Gawedzki, A. Kupiainen, Phys. Lett. B215 (1988) 119; Nucl. Phys. B320 (1989) 625; D. Karabali, Q.-H. Park, H. J. Schnitzer, Z. Yang, Phys. Lett. B216 (1989) 307; H. J. Schnitzer, Nucl. Phys. B324 (1989) 412; D. Karabali, H. J. Schnitzer, Nucl. Phys. B329 (1990) 649.

[7] J. Wess, B. Zumino, Phys. Lett. B37 (1971) 95; E. Witten, Comm. Math. Phys. $\underline{92}$ (1984) 455.

[8] K. Bardakci, M. Crescimanno, E. Rabinovici, Nucl. Phys. B344 (1990) 344; K. Bardakci, M. Crescimanno, S. Hotes, Nucl. Phys. B349 (1991) 439. 
[9] E. Witten, "On String Theory and Black Holes", IAS preprint, IASSNSHEP-91/12, March 1991.

[10] L. Dixon, J. A. Harvey, C. Vafa, E. Witten, Nucl. Phys. B261 (1985) 620; ibid. B274 (1986) 285.

[11] M. B. Halpern, J. P. Yamron, Nucl. Phys. B351 (1991) 333.

[12] M. B. Halpern, E. Kiritsis, Mod. Phys. Lett. A4 (1989) 1373;Erratum ibid. A4 (1989) 1797; A. Morozov, A. Perelomov, A. Rosly, M. Shifman, A. Turbiner, Int. J. Mod. Phys. A5 (1990) 803; M. B. Halpern, E. Kiritsis, N. A. Obers, M. Porrati, Int. J. Mod. Phys. A5 (1990) 2275;

[13] K. Kikkawa, M. Yamasaki, Phys. Lett. B149 (1984) 357; N. Sakai, I. Senda, Prog. Theor. Phys. $\underline{75}$ (1986) 692; V. P. Nair, A. Shapere, A. Strominger, F. Wilczek, Nucl. Phys. B287 (1987) 402; T. H. Buscher, Phys. Lett. B201 (1988) 466; A. Giveon, E. Rabinovici, G. Veneziano, Nucl. Phys. B322 (1989) 167; A. Shapere, F. Wilczek, Nucl. Phys. B320 (1989) 669; A. Giveon, N. Malkin, E. Rabinovici, Phys. Lett. B220 (1989) 551; ibid. B238 (1990) 57.

[14] A. Giveon, "Target Space Duality and Stringy Black Holes", Berkeley preprint, LBL-30671, April 1991; A. A. Tseytlin, "Duality and the Dilaton", Johns Hopkins preprint, JHU-TIPAC-91008, April 1991.

[15] S. Elitzur, A. Forge, E. Rabinovici, "Some Global Aspects of String Compactifications", Hebrew U. preprint, RI-143/90, Jan. 1991; G. Mandal, A. M. Sengupta, S. R. Wadia, "Classical Solutions of 2-d String Theory", IAS Preprint, IASSNS-HEP/91/10, March 1991;A. R. Lugo, J. Russo, "Black Holes in c=1 Liouville Gravity", Stanford preprint, SU-ITP-896, April 1991.

[16] N. J. Hitchin, A. Karlhede, U. Lindström, M. Roček, Comm. Math. Phys. $\underline{108}$ (1987) 535. 
[17] E. Martinec, S. Shatashvili, "Black Hole Physics and Liouville Theory", EFI preprint, EFI-91-22, May 1991.

[18] R. Dijkgraaf, E. Verlinde, H. Verlinde, "String Propagation in a Black Hole Geometry", IAS preprint, IASSNS-HEP-91/22, May 1991. 